\title{
44206 - A CLINICAL COMPARISON OF DOUBLE LUMEN TUBES AND THREE DESIGNS OF BRONCHIAL BLOCKERS FOR LUNG ISOLATION
}

\author{
Manu Narayanaswamy, Toronto General Hospital, Toronto, ON, Canada; \\ Slinger Peter, Toronto General Hospital; \\ McRae Karen, Toronto General Hopsital; \\ G Kanellakos, Toronto General Hospital; \\ A Roscoe;
}

Studies suggest that bronchial blockers (BBs) may be equivalent choices to double-lumen tubes (DLTs) for lung isolation in thoracic surgery .1 Recently three new designs of BB have become available the Arndt, the Cohen and the Fuji. This study compared these 3 BBs with DLTs to determine whether there were clinical advantages of one over the other during one-lung ventilation (OLV).

Fifty-four patients having left-sided thoracic or esophageal procedures were randomly assigned to one of four groups. Local IRB approval was obtained for this study.

The following were documented: 1) Time to endotracheal intubation; 2) Time required to position each tube/blocker 3) Number of attempts at placing the lung isolation device; 4) Frequency of intra-operative re-positioning. 5) Intra-operative lung isolation problems; 6) Lung collapse scores ranked by surgeons blinded to type of tube used; and 6) Time to complete surgery.

DLTs needed to be repositioned $7.7 \%$ of the times and the BB needed to be repositioned to $37.5 \%$ of the times. There was no statistical difference between the Fuji, Cohen and Arndt BB in the need to reposition them during the case. There was a significant difference between the DLTs and BBs for lung isolation problems during the case (DLTs $0 \%$ versus BB 37.5\%; $\mathrm{p}<0.01$ ). No difference was found between the three BBs. The time to lung isolation was significantly different between the DLT mean $93.8 \mathrm{~s}$ versus BB $250 \mathrm{~s}(\mathrm{p}<0.001)$. There was no statistical difference in the percentage of lung isolation devices that were placed during the first attempt (DLT $78.6 \%$ versus $60.5 \% \mathrm{BB}$ ). There was no difference between the different BBs in the percentage that were placed on first attempt. There was no correlation with respect to the angle the Left Main bronchus (LMB) to the trachea and the difficulty in placing the lung isolation device. The lung deflation scores ( 0 No lung collapse to 10 perfect lung collapse) were significantly different at chest opening DLT mean score 7.1 versus BB $4.8(\mathrm{p}=0.014)$. There were no differences in the lung deflation scores at 10, and 20 minutes after chest opening. There was no significant effect in this analysis of data to suggest that either early or late suction applied to the unventilated lung made any difference to the lung deflation score. 
We conclude that there were more intra-operative problems with BBs, irrespective of the design, compared to DLTs. After 10 min of chest opening, lung collapse was equivalent between BBs and DLTs.

1. Campos JH, Hallam EA, Van Natta T, Kernstine KH: Device for lung isolation used by anesthesiologists with limited thoracic experience: Comparison of double-lumen endotracheal tube, Univent ${ }^{\circledR}$ torque control blocker, and Arndt wire-guided endobronchial blocker®. Anesthesiology 2006; 104:261-6 